\title{
Serum proteomics and disease-specific biomarkers of patients with advanced gastric cancer
}

\author{
HELGI H. HELGASON ${ }^{1}$, JUDITH Y.M.N. ENGWEGEN ${ }^{1,3}$, MARK ZAPATKA ${ }^{5}$, ANNEMIEKE CATS ${ }^{2}$, \\ HENK BOOT $^{2}$, JOS H. BEIJNEN ${ }^{3,4}$ and JAN H.M. SCHELLENS ${ }^{1,4}$
}

\begin{abstract}
Divisions of ${ }^{1}$ Clinical Pharmacology and ${ }^{2}$ Gastroenterology and Hepatology, Department of Medical Oncology, The Netherlands Cancer Institute, Antoni van Leeuwenhoek Hospital, Amsterdam; ${ }^{3}$ Department of Pharmacy and Pharmacology, The Netherlands Cancer Institute, Slotervaart Hospital, Amsterdam; ${ }^{4}$ Division of Drug Toxicology, Section of Biomedical Analysis, Department of Pharmaceutical Sciences, Faculty of Science, Utrecht University, Utrecht, The Netherlands; ${ }^{5}$ Department of Theoretical Bioinformatics, German Cancer Research Center, Heidelberg, Germany
\end{abstract}

Received September 1, 2009; Accepted November 3, 2009

DOI: 10.3892/ol_00000058

\begin{abstract}
Gastric cancer is a commonly diagnosed solid tumor which is associated with a dismal prognosis making early diagnosis essential. Thus, this study aimed to identify novel biomarkers in gastric cancer. Serum of patients with advanced gastric cancer was collected according to a predefined schedule: prior to first-line chemotherapy with epirubicin $\left(50 \mathrm{mg} / \mathrm{m}^{2}\right.$, day 1$)$, cisplatin $\left(60 \mathrm{mg} / \mathrm{m}^{2}\right.$, day 1$)$ and capecitabine $\left(1,000 \mathrm{mg} / \mathrm{m}^{2}\right.$, twice daily on days $\left.1-14\right)$. The serum was collected serially before the treatment cycles and then analyzed by SELDI-TOF MS. Normal control subjects were matched according to age, gender and serum collection. Serum proteomic mass spectrometry data of all subjects were processed using the tbimass R-package and compared. We analyzed i) whether proteomic profile changes were associated with a response to chemotherapy and survival, and ii) whether changes in proteomic profiles occurring during the time period of chemotherapy were associated with tumor response. In total, 82 patients with adenocarcinoma of the stomach (mean age 57 years, males $69.5 \%$ ) were treated with a mean number of five chemotherapy cycles. The overall tumor response rate, complete and partial remission combined, was $37 \%$, median time to progression was 7 months (95\% CI, 6-8) and median overall survival 11 months (95\% CI, 9.5-12). By comparing 77 serum samples of patients with normal matched controls, we identified 32 proteins which discriminated the two groups. By selecting the most differentiating proteins, we built a classification model that correctly categorized $81 \%$ of the gastric cancer patients and $90 \%$ of the normal controls.
\end{abstract}

Correspondence to: Dr H.H. Helgason, Division of Clinical Pharmacology, Department of Medical Oncology, The Netherlands Cancer Institute, Antoni van Leeuwenhoek Hospital, Plesmanlaan 121, 1066 CX Amsterdam, The Netherlands

E-mail: h.helgason@nki.nl

Key words: proteomic, profile, gastric cancer, biomarker, prognostic
Furthermore, we found a statistically significant correlation between the pre-treatment intensity of serum amyloid- $\alpha$ (SAA) and overall survival in gastric cancer patients, whereby a low intensity of SAA predicted a longer patient survival. A classification model, based on the 32 most discriminating proteins differentiating gastric cancer from normal controls, correctly classified subjects with relatively high sensitivity and specificity.

\section{Introduction}

Despite a declining incidence of distal gastric cancer, the incidence of adenocarcinoma of the distal esophagus and stomach has increased and remains among the most common malignancies in the world and the second leading cause of cancer-related death (1). There exists marked geographical variation in incidence with higher occurrence in Asia compared to Europe and the US (2). In the western world most patients with esophageal or gastric cancer present at a late stage with locally advanced or metastatic disease beyond curative options. Only in a minority of patients presenting at an early stage, does surgical resection have a real curative intent resulting in 5-year survival rates of approximately $70 \%$ for stage I, while in stage II the 5-year survival drops to only $35 \%$ (3). Patients with advanced adenocarcinoma of the distal esophagus or stomach have a dismal prognosis making early detection of the utmost importance $(4,5)$. Although screening with gastroscopy has been used in endemic areas, this approach has limitations regarding patient burden, accuracy, availability and cost (6,7). In developed countries, the prognosis of patients with solid malignancies has improved gradually. The use of tailored surgery, sophisticated radiotherapy and the use of adjuvant medical treatment in breast, colorectal and more recently gastric cancer have increased significantly. Both postoperative chemoradiotherapy and perioperative chemotherapy are associated with better disease-free and overall survival in gastric cancer $(8,9)$. Concomitantly, with an improved prognosis, the early costs of treatment have increased significantly. Moreover, the treatment of metastatic 
disease with novel agents is an increasingly costly undertaking. Therefore, in light of the increased cost of health care, it is of major importance to find easily applicable and robust technology that improves the early detection of malignancy, and predicts treatment response and patient survival.

Serum biomarkers within the proteome are among the more promising future screening tools for cancer detection, as prognostic markers for disease relapse and survival and potentially as predictive markers of chemotherapy response. Surface-enhanced laser desorption/ionization-time of flight (SELDI-TOF) mass spectrometry (MS) technology enables the analysis of the relative expression levels of proteins over a wide range of molecular weights in biological samples, focusing on low abundant proteins. Differences in serum protein expression levels may be used to identify disease-specific proteomic profiles or 'fingerprints'. Proteomic analysis potentially also avoids overlooking posttranslational modifications and may be a useful method in the analysis of changes occurring over time during or following chemotherapy (10).

Previously, our group identified several candidate biomarkers for renal cell carcinoma by using the same proteomic analytical technique. Although some $\mathrm{m} / \mathrm{z}$ values were difficult to reproduce, the increased expression of the previously identified serum amyloid- $\alpha$ (SAA) peak cluster was validated in different patient populations (11).

We hypothesized that we would be able to identify i) novel and disease-specific peptides that differentiate patients with advanced adenocarcinoma of the distal esophagus or stomach (GC) from normal controls (NC), ii) peptide profiles that would be able to predict response or prolonged survival following palliative chemotherapy, and iii) peptides that change differentially over time during chemotherapy in chemotherapy responsive and non-responsive patients. Serum of GC patients was prospectively collected, prior to and during first-line chemotherapy with epirubicin, cisplatin and capecitabine, and simultaneously from matched $\mathrm{NC}$, and then analyzed by SELDI-TOF MS.

\section{Materials and methods}

Patient characteristics (Table I). Serum samples were prospectively collected from all chemotherapy naive patients with histologically confirmed advanced adenocarcinoma of the distal esophagus or stomach (GC). The study was approved by the local medical ethics committee, and the patients gave written informed consent. Only patients with a performance score $\mathrm{WHO} \leq 2$, measurable disease according to RECIST criteria (12) and adequate haematological, renal and hepatic functions [absolute neutrophil count $\geq 1.5 \times 10^{9} / 1$, platelets $\geq 100 \times 10^{9} / 1$, bilirubin $\leq 1.5$ times the upper limit of the normal range (ULN), AST and ALT $\leq 2.0 \mathrm{ULN}$, but in the presence of liver metastases $\leq 5.0 \mathrm{ULN}$; serum creatinine $\leq 2.0$ times ULN] were eligible. Previous surgery was allowed. The control group consisted of normal subjects that were selected based on a short questionnaire and matched for age, gender and time period of blood donation.

Treatment and tumor evaluation. Patients received firstline chemotherapy with epirubicin $\left(50 \mathrm{mg} / \mathrm{m}^{2}\right)$ and cisplatin $\left(60 \mathrm{mg} / \mathrm{m}^{2}\right)$ intravenously on day 1 , followed by oral capecit-
Table I. Patient characteristics.

\begin{tabular}{lcr}
\hline \multirow{2}{*}{ Characteristic } & \multicolumn{2}{c}{ Patients } \\
\cline { 2 - 3 } & No. & $\%$ \\
\hline Total & 82 & 100 \\
Median age (range) & $57(34-74)$ \\
Gender & & \\
Male & 57 & 70 \\
Female & 25 & 30 \\
Prior treatment & & \\
Chemoradiotherapy & 1 & 1 \\
Radiotherapy (>6 months prior to inclusion) & 8 & 11 \\
Primary site & & \\
Distal esophagus or gastric cardia & 45 & 55 \\
Sites of metastases & & \\
Lymph nodes & 71 & 87 \\
Abdominal cavity & 30 & 37 \\
Liver & 21 & 26 \\
Lungs & 7 & 9 \\
Bones & 3 & 4 \\
$\geq 2$ Metastatic sites & 48 & 57 \\
\hline & &
\end{tabular}

Table II. Treatment characteristics.

\begin{tabular}{cc}
\hline No. of treatment cycles & No. of patients \\
\hline $0-1$ & 5 \\
$2-4$ & 31 \\
$5-6$ & 41 \\
$>6$ & 5 \\
\hline
\end{tabular}

abine $\left(1,000 \mathrm{mg} / \mathrm{m}^{2}\right)$ twice daily on days $1-14$ (ECC), every 3 weeks (Table II). Tumor response was assessed every other cycle by computer tomography scan.

Sample collection and definitions. Whole blood samples were obtained at regular predefined times: intervals starting prior to the start of chemotherapy and immediately prior to each chemotherapy cycle in weeks $3,6,9,12,15$ and 18 or later in case of treatment delay. Whole blood samples of patients and normal controls were collected by applying a standardized drawing and handling procedure in standard tubes (BD Vacutainer $^{\mathrm{TM}}$ SST II $8.5 \mathrm{ml}$; BD Company, Franklin Lakes, NJ, USA). Samples were allowed to clot for $15 \mathrm{~min}$ and then centrifuged at 3,000 rpm for $10 \mathrm{~min}$ at room temperature (13). Subsequently, the serum was transferred in equal aliquots to five polypropylene tubes $(1.4 \mathrm{ml})$ and stored at $-30^{\circ} \mathrm{C}$ until analysis. The serum samples originated from the Netherlands Cancer Institute serum bank.

The primary analysis consisted of a comparison of the proteomic profiles of GC patients and NC subjects. For the subsequent analysis of proteomic profile differences between 
responding patients and non-responders, the patients were divided into two groups according to response: i) responder: patients developing complete response, partial response and stable disease for a duration of $>6$ months, respectively, and ii) non-responders: patients developing stable disease for a duration of $<6$ months or progressive disease, respectively. In the search for a prognostic proteomic profile for predicting survival, we divided the patients according to $\leq$ or $>6$-month survival.

SELDI-TOF analysis. Protein profiling was performed using SELDI-TOF MS (Biorad Laboratories, Hercules, CA, USA). Previously, we screened different chromatographic and binding conditions in patients with colorectal cancer (14). The CM 10 chip is a weak cation exchange chip that contains anionic carboxylate groups that bind positively charged proteins in serum. A binding buffer of $20 \mathrm{mM}$ sodium phosphate $+0.1 \%$ Triton X-100 (Sigma, St. Louis, MO, USA) (pH 5.0) and a 100\% solution of sinapinic acid (SPA; BioRad Laboratories) in $50 \%$ acetonitrile $+0.5 \%$ trifluoracetic acid as an energy absorbing matrix yielded the most discriminating $\mathrm{m} / \mathrm{z}$ values (12).

Samples were thawed only once and analyzed twice (in doublets). After thawing, the serum samples were denatured by adding $180 \mu \mathrm{l}$ of a solution containing $9 \mathrm{M}$ urea, $2 \%$ CHAPS, $1 \%$ DTT (all from Sigma) to $20 \mu \mathrm{l}$ of serum. CM 10 chips were assembled in 96-well format bioprocessors (BioRad Laboratories). During all steps of the protocol, the bioprocessor was placed on a platform shaker at $350 \mathrm{rpm}$. Chips were equilibrated twice with $200 \mu \mathrm{l}$ of binding buffer for $5 \mathrm{~min}$. Subsequently, $180 \mu \mathrm{l}$ of binding buffer and $20 \mu \mathrm{l}$ of denatured sample were applied to the chip surface. Sample allocation was random for comparison of GC vs. NC sera. For the analysis of serial GC sera, all samples from the same patient were analyzed on the same chip whenever possible, and the remaining samples were allocated at random. For quality control, a separate sample from a healthy volunteer was used and spotted on the remaining locations (4-6 spots) across the bioprocessor. Incubation was set to $30 \mathrm{~min}$. After binding, the chips were washed twice for 5 min with binding buffer, followed by two 5-min washings with binding buffer without Triton X-100. Finally, the chips were rinsed with deionised water, air dried and finished with two 1- $\mu 1$ SPA applications to the sample spots. The reproducibility of the applied methodology was previously validated by our group (11).

Protein chips were analyzed using the PBS-IIC Protein Chip Reader (BioRad Laboratories). Data were collected between 0 and 200,000 Da. Data collection was optimized for detection of discriminating peaks, resulting in an average of 65 laser shots per spectrum at laser intensity 150 , detector sensitivity 8 and laser focusing at 3,000 $\mathrm{Da}$. M/z values for the detected proteins were calibrated externally with a standard peptide mixture (BioRad Laboratories) containing vasopressin $(1,084.3 \mathrm{Da})$, somatostatin (1,637.9 Da), dynorphine (2,147.5 Da), ACTH (2,933.5 Da), insulin $\beta$-chain (bovine; 3,495.5 Da), insulin (human recombinant; 5,807.7 Da) and hirudin (7,033.6 Da) (11).

Bioinformatics. Serum proteomic MS data of GC patients and matched $\mathrm{NC}$ were processed using the tbimass R-package

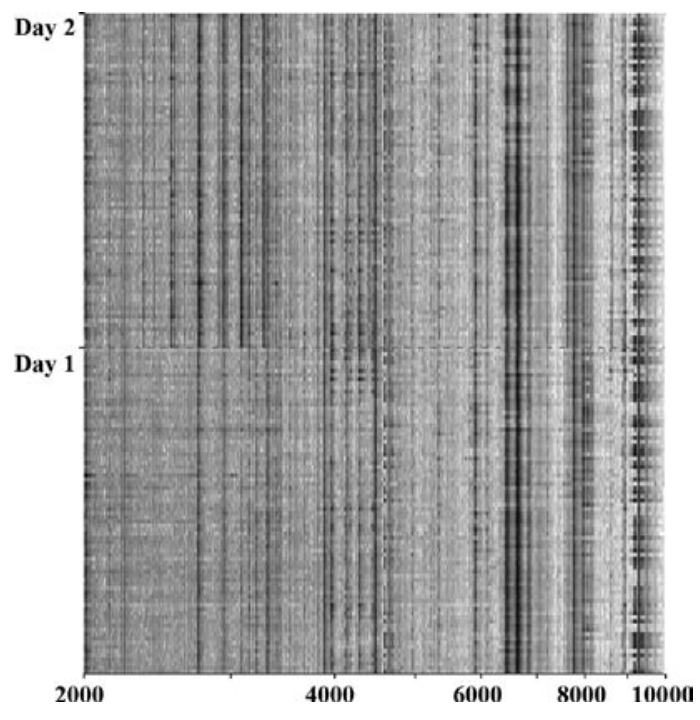

Figure 1. Differences in the proteomic pattern due to day-to-day variation.

(www.r-project.org). For pre-processing, the spectra were re-sampled to a common $\mathrm{m} / \mathrm{z}$ vector, and the baseline was corrected using the PROcess R-Package. Furthermore, the intensity of the spectra was normalised to the total ion current to reduce noisy variance between replicate measurements (15). To correct for small deviations in the $\mathrm{m} / \mathrm{z}$ values due to calibration, the alignment algorithm by Jeffries was implemented in tbimass and applied (16). For classification, the support vector machine implementation within the MCRestimate R-package was applied. For variable selection, a variable filtering procedure based on the relative intensity variance was used for classification. To assess the classification accuracy, a 10-fold repetition of 10 -fold cross validation with a nested 3 -fold parameter optimisation loop was conducted. The number of variables used for classification was reduced in each classification by recursive feature elimination (17).

\section{Results}

Clinical outcome. A total of 82 patients with adenocarcinoma of the distal esophagus and stomach were treated with first-line chemotherapy (Tables I and II). The mean age was 57 years (range 34-74) and there were 57 males $(69.5 \%)$ and 25 females (30.5\%). Patients were previously untreated except one patient who had received chemoradiotherapy, including capecitabine, and 8 patients who had received radiotherapy for proximal gastric carcinoma, respectively. The patients had locally advanced or metastatic gastric cancer and were therefore all included in the survival analysis and the proteomic profiling. Fourteen patients were not assessable for response according to RECIST criteria (12). Seven patients had only localized disease and were operable after chemotherapy, and 7 other patients were excluded after radiological review of CT scans due to non-measurable disease. The mean follow-up was 12 months, and the mean number of chemotherapy cycles was five. Complete and partial response was noted in 5 and 20 patients, respectively (response rate, 37\%; intention to treat, $30 \%$ ). Additionally, 38 patients had stable disease for $>3$ months; 18 of these for $>6$ months. Five patients had 
Table III. Objective response rate according to RECIST criteria.

\begin{tabular}{lcc}
\hline Response & No. of patients & $\%$ \\
\hline Evaluable for proteomics & 78 & 95 \\
Evaluable for response & 68 & 83 \\
Complete response & 5 & 7 \\
Partial response & 20 & 30 \\
Stable disease & 38 & 56 \\
$\quad(>6$ months) & $(18)$ & $(26)$ \\
Progressive disease & 5 & 7 \\
\hline
\end{tabular}

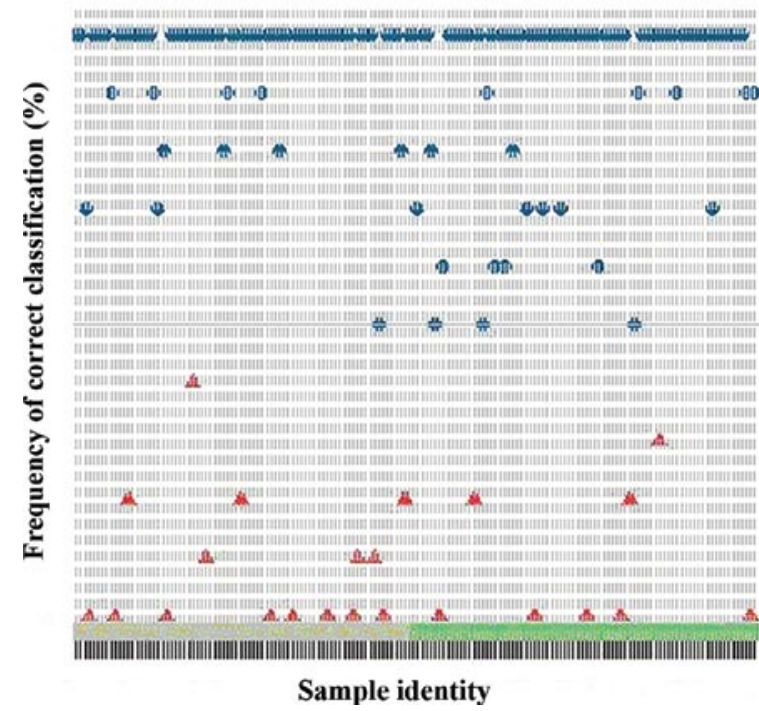

Figure 2. Frequency of correct classification (GC, blue; NC, red).

chemotherapy-resistant disease and showed progression at the time of first evaluation. The median time to progression was 6.2 months (95\% CI, 5.6-6.7), median progression-free survival was 6 months (95\% CI, 5.4-6.5) and median overall survival was 10.8 months (95\% CI, 9.5-12.1), respectively. In case of progression, the most common sites were local lymph nodes, peritoneal cavity, liver and bones.

Proteomic profiling of gastric cancer patients and normal controls. Serum obtained immediately prior to the start of ECC chemotherapy in all 82 patients with advanced or metastatic GC was analyzed by SELDI-TOF MS and compared with serum of $80 \mathrm{NC}$. Patients were matched for age, gender and time-period of serum collection. In the pre-processing normalization procedure, 4 serum samples from the GC population were categorized as outliers and excluded from further analysis (13). By global proteomic profiling, some differences in the proteomic profile of GC patients, according to the day of SELDI-TOF analysis, was noticed (Fig. 1). By comparing GC patients and $\mathrm{NC}$ we identified $32 \mathrm{~m} / \mathrm{z}$ values that differentiated between the two groups (Table III). Fourteen of these were identified during the first measurement run 1 , and 19 during the second measurement performed one day later.
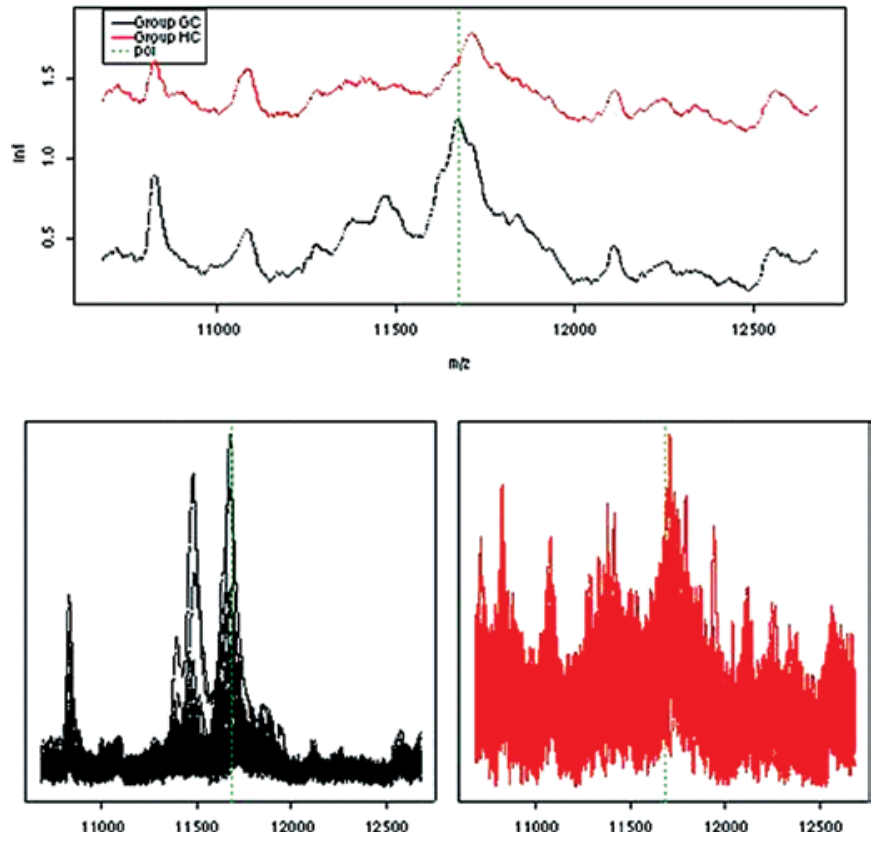

Figure 3. Protein intensity of peak $11.6 \mathrm{kDa}$ (serum amyloid- $\alpha$ ).

One $\mathrm{m} / \mathrm{z}$ value was identified by the two measurements. To minimize the influence of day-to-day variations, we based the further classification on the proteomic profiling of all serum samples, independent of the day of measurement. The quality of the classification model was not influenced by the difference in identity and intensity of the most discriminating proteins for GC and NC. The classification model built on the pooled dataset correctly classified 72 out of the $80 \mathrm{NC}$ (specificity, 90\%) and 63 out of the 78 GC patients (sensitivity, 81\%) (Fig. 2).

Proteomic profiling and response prediction. Proteomic profiles of serum obtained from GC patients immediately prior to the start of ECC chemotherapy were analyzed according to response to chemotherapy. Response evaluation was determined prior to the start and after every second cycle, according to the protocol, in 68 patients eligible for response evaluation. Patients were divided into two groups: responders (43) and non-responders (25), respectively. By applying the MannWhitney U test, a positive correlation was observed between six proteomic peaks $(3.0,3.1,3.8,4.7,7.6$ and $33.3 \mathrm{kDa})$ and treatment response, but none significantly predicted chemotherapy effect.

Proteomic profiling and survival prediction. Proteomic profiles of serum obtained from GC patients immediately prior to the start of ECC chemotherapy were related to overall survival. Median overall survival of the patients was 11 months (95\% CI, 9.5-12). Using data dichotomisation and multivariate Cox regression analysis, a significant positive relationship was observed between low intensity (cut-off value $<0.4$ ) of the protein $\mathrm{m} / \mathrm{z} 11.6 \mathrm{kDa}$ and longer survival of $12 \mathrm{vs}$. 9.6 months, respectively $(\mathrm{p}=0.003)$. This $\mathrm{m} / \mathrm{z}$ value has been shown to be SAA with a molecular weight of $11.6 \mathrm{kDa}$. In concordance, a higher expression of SAA has previously been correlated with advanced malignancies (18) and various forms 
Table IV. The most important $\mathrm{m} / \mathrm{z}$ values contributing to the classification model according to the day of SELDI-TOF analysis.

\begin{tabular}{|c|c|}
\hline Peak (Da) & Importance \\
\hline \multicolumn{2}{|l|}{ Day $1^{\mathrm{b}}$} \\
\hline 3892.7447 & 0.714944646 \\
\hline 15625.932 & 0.675882146 \\
\hline 29686.354 & 0.657327458 \\
\hline 9989.2487 & 0.594808005 \\
\hline 10574.214 & 0.589944646 \\
\hline 46048.839 & 0.588948630 \\
\hline 6674.9972 & 0.587991521 \\
\hline 144017.79 & 0.576272771 \\
\hline 7096.0364 & 0.570413396 \\
\hline 3775.4055 & 0.562600896 \\
\hline 8291.9817 & 0.560596815 \\
\hline 4438.7837 & 0.550882146 \\
\hline 9721.6843 & 0.548929021 \\
\hline 124829.75 & 0.547933005 \\
\hline \multicolumn{2}{|l|}{ Day $2^{c}$} \\
\hline 3892.745 & 1.459401 \\
\hline 40544.12 & 1.200624 \\
\hline 6623.487 & 1.141141 \\
\hline 13736.36 & 1.048368 \\
\hline 4245.927 & 1.034613 \\
\hline 24024.73 & 0.868681 \\
\hline 3639.509 & 0.783559 \\
\hline 4548.882 & 0.768973 \\
\hline 3316.726 & 0.621440 \\
\hline 25482.25 & 0.608915 \\
\hline 15618.04 & 0.603906 \\
\hline 25270.93 & 0.577259 \\
\hline 4641.661 & 0.556181 \\
\hline 4751.328 & 0.544462 \\
\hline 4377.272 & 0.544187 \\
\hline 25100.50 & 0.536512 \\
\hline 3891.431 & 0.534280 \\
\hline 2183.326 & 0.524931 \\
\hline 20841.38 & 0.505400 \\
\hline
\end{tabular}

aRelative importance of the peak value in the classification between

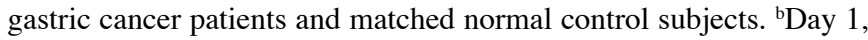
measurements performed on day 1 . $^{\mathrm{c}}$ Day 2 , measurements performed on day 2.

of acute phase reactions (19). These results correlate well with the proteomic profiling of the NC subjects who had the lowest median intensity of SAA (Fig. 3).

Serial proteomic profiling. Fifty patients with measurable disease according to RECIST had adequate serial sample collections at baseline prior to chemotherapy and sequentially thereafter at approximately 6,12 and 18 weeks after the start of the treatment. These serially collected serum samples were analyzed according to the best tumor response, which frequently developed after four treatment cycles. No significant proteomic changes or potential biomarkers associated with therapy monitoring were detected.

\section{Discussion}

In this single institutional phase II study, we described the detection of significantly different proteomic patterns in GC patients vs. NC subjects serving as potential biomarkers of gastric cancer. Protein analysis of serum from cancer patients by advanced technologies, including SELDI-TOF MS, is a promising tool with which to identify novel proteins, protein fragments or proteomic profiles that are specific for particular malignancies for use as biomarkers for disease detection, as prognostic parameters or for the prediction of treatment response.

The age distribution and metastatic pattern revealed that the patients selected most likely represent a real life population of GC patients. The median time to progression was 7 months and overall survival was 11 months, which is comparable to other studies of anthracycline, cisplatin and 5-fluorouracil chemotherapeutic combination regimens in patients with advanced gastric cancer (20). The study was conducted according to a strict protocol regarding serum collection, handling and storage at $-30^{\circ} \mathrm{C}$ to minimize pre-analytic influence of serum sampling on the SELDI-TOF protein profiles (21). The selected patients had advanced gastric adenocarcinoma and underwent standard first-line chemotherapy. This strategy enabled us to focus on a homogeneous patient population acknowledging the usual variability between different patient populations that may affect outcome of the analysis. Samples were analyzed twice on two separate days after sample preparation. By comparing GC and NC subjects, we identified 32 different $\mathrm{m} / \mathrm{z}$ values, representing peptides and proteins, which possibly correlate to the active malignant process, metastatic disease or survival. By pooling all of the most important $\mathrm{m} / \mathrm{z}$ values differentiating GC patients and $\mathrm{NC}$ subjects, the potential bias caused by differences in the outcome of proteomic analysis on different analysis days was limited. This made the analysis time-independent to the greatest extent possible. Selection of the included $\mathrm{m} / \mathrm{z}$ values was based on their relative importance of the peak value in the classification between GC patients and matched NC (Table IV). The classification model based on the whole dataset correctly classified GC patients and NC with $90 \%$ specificity and $81 \%$ sensitivity. This classification served as a potential GC-specific proteomic profile differentiating GC patients from normal subjects. A correlation analysis of proteomic profile vs. clinical outcome, response and survival, showed no predictive or prognostic biomarkers with any certainty, although we identified protein $11.6 \mathrm{kDa}$ as a potential prognostic biomarker. This $\mathrm{m} / \mathrm{z}$ value was previously identified as SAA with a molecular weight of $11.6 \mathrm{kDa}$. In concordance, a higher expression of SAA was previously related to advanced malignancies (18) and various types of acute phase reactions (19) and the finding was therefore anticipated. We were not able to identify reliable or predictive biomarkers of treatment response even though the sample size was relatively large. 
Possibly this was related to the intrinsic differences between the malignancies, differences in disease extent and differences in patient characteristics, such as gender and age. The influence of patient demographics on the proteomic profile is not well known, but Villanueva et al found that gender and age had negligible influence on the discrimination between patients with thyroid cancer and healthy subjects (22). In concordance with the inability to identify predictive biomarkers, in the serial analysis of 50 patients with gastric cancer, with consistent sample collection and response evaluation throughout the entire treatment, no significant changes in the predictive proteomic profile during chemotherapy were identified. In proteomic profiling studies in gastric cancer of comparable sample size and design performed by others, changes could not be identified. Several studies analyzing disease-specific proteomic patterns, in search of novel diagnostic or predictive biomarkers in advanced and early breast cancer patients have been published $(23,24)$. Irrespective of the different clinical setting and technical approach, most of these analyses found an association between several known proteins or their fragments, such as diverse apolipoproteins, complement factors, fibrinogen and haptoglobin. Many of these have been shown not to be disease-specific, and none have been validated in a prospective clinical study (25).

The most differentiating proteins, based on their relative importance of the peak value in the classification between gastric cancer patients and matched normal control subjects (Table IV), varied on different days of analysis. To further reduce any variability, analysis of all samples needs to be carried out on one single day. Many peptides detected by MS have not been structurally identified. Although a further characterization of the peptides included in the profile may help to understand the biological processes they represent, identification is not a prerequisite for the use of the profile for predictive or prognostic biomarkers. Our results need further validation in a prospective study in order to explore the reproducibility of the identified classifiers that may serve as biomarkers of gastric cancer.

One limitation of our study was the manual handling of the samples and the preparation of the samples for SELDI analysis after thawing. This is possibly the most important cause of the identified differences in the proteomic profiles on days 1 and 2 of the analysis. Automatic sample handling using robot systems will allow a much faster analysis and near simultaneous massspectrum analysis of complete sample sets eliminating the confounding effect of manual sample handling.

In conclusion, the identified proteomic profile enabled the differentiation between GC patients with advanced disease and NC subjects. We identified 32 protein peaks differentiating gastric cancer and normal controls that made it possible to build a classification model separating these two groups with a relatively high specificity and sensitivity. By incorporating strict sample handling, storage and analyses, we improved the robustness of SELDI-TOF MS analysis, but by introducing automatic robot sample handling methods, further optimization of proteomic profiling of solid malignancies may be possible. Future studies aimed at identifying surrogate proteomic profiles as prognostic biomarkers of gastric cancer and patient survival and predictive biomarkers of treatment efficacy are warranted.

\section{Acknowledgements}

Author contributions: Helgi H. Helgason and Jan H.M. Schellens designed the research. Helgi H. Helgason, Annemieke Cats, Henk Boot and Jan H.M. Schellens conducted the study. Helgi H. Helgason analyzed the clinical data. Helgi H. Helgason, Judith Y.M.N. Engwegen, Jos H. Beijnen and Mark Zapatka analyzed the proteomic results. Helgi H. Helgason, Jan H. Schellens and Judith Y.M.N. Engwegen wrote the manuscript.

\section{References}

1. Jemal A, Siegel R, Ward E, Hao Y, Xu J and Thun MJ: Cancer statistics 2009. CA Cancer J Clin 59: 225-249, 2009.

2. Parkin DM, Bray F, Ferlay J and Pisani P: Global cancer statistics, 2002. CA Cancer J Clin 55: 74-108, 2005.

3. Hundahl SA, Phillips JL and Menck HR: The National Cancer Data Base Report on poor survival of US gastric carcinoma patients treated with gastrectomy: Fifth Edition American Joint Committee on Cancer staging, proximal disease and the "different disease' hypothesis. Cancer 88: 921-932, 2000.

4. Karpeh MS, Leon L, Klimstra D and Brennan MF: Lymph node staging in gastric cancer: Is location more important than number? An analysis of 1,038 patients. Ann Surg 232: 362-371, 2000.

5. Lim L, Michael M, Mann GB and Leong T: Adjuvant therapy in gastric cancer. J Clin Oncol 23: 6220-6232, 2005.

6. Green PH, Fleischauer AT, Bhagat G, Goyal R, Jabri B and Neugut AI: Risk of malignancy in patients with celiac disease. Am J Med 115: 191-195, 2003.

7. Murakami R, Tsukuma H, Ubukata T, Nakanishi K, Fujimoto I, Kawashima T, Yamazaki $\mathrm{H}$ and Oshima A: Estimation of validity of mass screening program for gastric cancer in Osaka, Japan. Cancer 65: 1255-1260, 1990.

8. Macdonald JS, Smalley SR, Bendedetti J, Hundahl SA, Estes NC, Stemmermann GN, Haller DG, Ajani JA, Gunderson LL, Jessup JM and Martenson JA: Chemoradiotherapy after surgery compared with surgery alone for adenocarcinoma of the stomach or gastroesophageal junction. N Engl J Med 345: 725-730, 2001.

9. Cunningham D, Allum WH, Stenning SP, Thompson JN, van de Velde CJ, Nicolson M, Scarffe JH, Lofts FJ, Falk SJ, Iveson TJ, Smith DB, Langley RE, Verma M, Weeden S, Chua YJ and MAGIC Trial Participants: Perioperative chemotherapy versus surgery alone for resectable gastroesophageal cancer. $\mathrm{N}$ Engl J Med 355: 11-20, 2006.

10. Petricoin EF and Liotta LA: SELDI-TOF-based serum proteomic pattern diagnostics for early detection of cancer. Curr Opin Biotechnol 15: 24-30, 2004.

11. Engwegen JY, Mehra N, Haanen JB, Bonfrer JM, Schellens JH, Voest EE and Beijnen JH: Validation of SELDI-TOF MS serum protein profiles for renal cell carcinoma in new populations. Lab Invest 87: 161-172, 2007.

12. Therasse P, Arbuck SG, Eisenhauer EA, Wanders J, Kaplan RS, Rubinstein L, Verweij J, van Glabbeke M, van Oosterom AT, Christian MC and Gwyther SG: New guidelines to evaluate the response to treatment in solid tumors. European Organization for Research and Treatment of Cancer, National Cancer Institute of the United States, National Cancer Institute of Canada. J Natl Cancer Inst 92: 205-216, 2000.

13. Engwegen JY, Gast MC, Schellens JH and Beijnen JH: Clinical proteomics: searching for better tumour markers with SELDI-TOF mass spectrometry. Trends Pharmacol Sci 27: 251-259, 2006.

14. Engwegen JY, Helgason HH, Cats A, Harris N, Bonfrer JM, Schellens $\mathrm{JH}$ and Beijnen $\mathrm{JH}$ : Identification of serum proteins discriminating colorectal cancer patients and healthy controls using surface-enhanced laser desorption ionisation-time of flight mass spectrometry. World J Gastroenterol 12: 1536-1544, 2006.

15. Meuleman W, Engwegen JY, Gast MC, Beijnen JH, Reinders MJ and Wessels LF: Comparison of normalisation methods for surface-enhanced laser desorption and ionisation (SELDI) timeof-flight (TOF) mass spectrometry data. BMC Bioinformatics 9: 88, 2008. 
16. Jeffries N: Algorithms for alignment of mass spectrometry proteomic data. Bioinformatics 21: 3066-3073, 2005.

17. Guyon I, Weston J and Barnhill S: Gene selection for cancer classification using support vector machines. Machine Learning 46: 389-422, 2002.

18. Maciel CM, Junqueira M, Paschoal ME, Kawamura MT, Duarte RL, Carvalho Mda G and Domont GB: Differential proteomic serum pattern of low molecular weight proteins expressed by adenocarcinoma lung cancer patients. J Exp Ther Oncol 5: 31-38, 2005.

19. Kosuge M, Ebina T, Ishikawa T, Hibi K, Tsukahara K, Okuda J, Iwahashi N, Ozaki H, Yano H, Kusama I, Nakati T, Umemura S and Kimura K: Serum amyloid A is a better predictor of clinical outcomes than C-reactive protein in non-ST-segment elevation acute coronary syndromes. Circ J 71: 186-190, 2007.

20. Wagner AD, Grothe W, Haerting J, Kleber G, Grothey A and Fleig WE: Chemotherapy in advanced gastric cancer: a systematic review and meta-analysis based on aggregate data. J Clin Oncol 24: 2903-2909, 2006

21. Timms JF, Arslan-Low E, Gentry-Maharaj A, Luo Z, T'Jampens D, Podust VN, Ford J, Fung ET, Gammerman A, Jacobs I and Menon U: Preanalytic influence of sample handling on SELDI-TOF serum protein profiles. Clin Chem 53: 645-656, 2007.
22. Villanueva J, Martorella AJ, Lawlor K, Philip J, Fleisher M, Robbins RJ and Tempst P: Serum peptidome patterns that distinguish metastatic thyroid carcinoma from cancer-free controls are unbiased by gender and age. Mol Cell Proteomics 5: 1840-1852, 2006.

23. Van Winden AW, Gast MC, Beijnen JH, Rutgers EJ, Grobbee DE, Peeters PH and van Gils CH: Validation of previously identified serum biomarkers for breast cancer with SELDI-TOF MS: a case control study. BMC Med Genomics 2: 4, 2009.

24. Li J, Orlandi R, White CN, Rosenzweig J, Zhao J, Seregni E, Morelli D, Yu Y, Meng XY, Zhang Z, Davidson NE, Fung ET and Chan DW: Independent validation of candidate breast cancer serum biomarkers identified by mass spectrometry. Clin Chem 51: 2229-2235, 2005

25. Gast MC, Schellens JH and Beijnen JH: Clinical proteomics in breast cancer: a review. Breast Cancer Res Treat 116: 17-29, 2009. 